\title{
Mikio Sato and Mathematical Physics
}

\author{
by
}

Barry M. McCoY

\begin{abstract}
I present the deep and lasting contributions of Mikio Sato to the mathematical physics of statistical mechanics and random matrix theory.

2010 Mathematics Subject Classification: 01A60.

Keywords: Ising model, statistical mechanics.

Prof. Mikio Sato is unique among Japanese mathematicians, and indeed, perhaps among all mathematicians, in that he was trained as a graduate student by a Nobel Prize winning physicist, Sin-Itero Tomonaga. This unique training has given Prof. Sato a deep insight into the relation of mathematics to physics and has led to the creation of profound mathematical tools for the solution of problems in statistical mechanics and random matrix theory. The discoveries of Prof. Sato have inspired an entire younger generation of Japanese mathematicians to enter the field of mathematical physics which has led to the invention of quantum groups and revolutionary advances in representation theory. In this note I will sketch the discoveries of Prof. Sato in mathematical physics and outline the scope of achievements of what may rightly be called the "Sato School" which have been inspired by his work and by his training.
\end{abstract}

\section{$\S 1$. The Ising model}

The Ising model is a system in two dimensions with variables (called spins) $\sigma_{j, k}=$ \pm 1 at the vertices of a rectangular lattice of $L_{v}$ sites in the vertical direction and $L_{h}$ sites in the horizontal direction which interact with each other with an

This is a contribution to the special issue "The golden jubilee of algebraic analysis".

Communicated by M. Kashiwara. Received December 22, 2009.

B. M. McCoy: C. N. Yang Institute for Theoretical Physics, State University of New York, Stony Brook, NY 11794-3840, USA;

e-mail: barry.mccoy@stonybrook.edu 
interaction energy

$$
\mathcal{E}=-\sum_{j=1}^{L_{v}} \sum_{k=1}^{L_{h}}\left\{E^{v} \sigma_{j, k} \sigma_{j+1, k}+E^{h} \sigma_{j, k} \sigma_{j, k+1}\right\}
$$

with periodic boundary conditions. The questions of interest to physicists are the computation of the partition function

$$
Z=\sum_{\sigma_{j, k}= \pm 1} \exp \left\{-\mathcal{E} / k_{B} T\right\}
$$

the free energy per site

$$
-F / k_{B} T=\lim _{L_{v}, L_{h} \rightarrow \infty}\left(L_{v} L_{h}\right)^{-1} \ln Z
$$

and the correlation functions

$$
\left\langle\sigma_{j_{1}, k_{1}} \cdots \sigma_{j_{n}, k_{n}}\right\rangle=\lim _{L_{v}, L_{h} \rightarrow \infty} Z^{-1} \sum_{\sigma_{j, k}= \pm 1} \sigma_{j_{1}, k_{1}} \cdots \sigma_{j_{n}, k_{n}} \exp \left\{-\mathcal{E} / k_{B} T\right\}
$$

where $\sum_{\sigma_{j, k}= \pm 1}$ is the sum over all states of the system, $T$ is the temperature and $k_{B}$ is Boltzmann's constant. The free energy (1.3) was computed by Onsager [8] in 1944, the partition function 1.2 for finite $L_{v}, L_{h}$ was computed by Kaufman [6] in 1949 and correlation functions were computed as determinants by Kaufman and Onsager [7] in 1949.

These computations of Onsager and of Kaufman are one of the major discoveries in mathematical physics of the 20th century and from these computations it is seen that there is a special value of the temperature $T_{c}$ defined as the solution of the equation

$$
\sinh \left(2 E^{h} / k_{B} T_{c}\right) \sinh \left(2 E^{v} / k_{B} T_{c}\right)=1
$$

where the free energy has a singularity of the form $\left(T-T_{c}\right)^{2} \ln \left|T-T_{c}\right|$.

It is of great interest to study the correlation functions $\left\langle\sigma_{j_{1}, k_{1}} \cdots \sigma_{j_{n}, k_{n}}\right\rangle$ in the vicinity of $T=T_{c}$. In particular it is of great interest to study the twopoint spin correlation function $\left\langle\sigma_{0,0} \sigma_{M, N}\right\rangle$ for $M$ and $N$ large. Unhappily the size of the determinants which give the correlation functions grows linearly with the separation between the spins.

The first result on the large separation behavior of the correlation functions is the computation of the order parameter $M_{-}$which may be defined as

$$
M_{-}^{2}=\lim _{M^{2}+N^{2} \rightarrow \infty}\left\langle\sigma_{0,0} \sigma_{M, N}\right\rangle
$$


This order parameter was announced by Onsager [9] in 1949 and proven by Yang [20] (in the isotropic case) to be

$$
M_{-}=\left(1-k^{2}\right)^{1 / 8}
$$

for $T<T_{c}$ and zero for $T \geq T_{c}$ where

$$
k=\left(\sinh \left(2 E^{h} / k_{B} T\right) \sinh \left(2 E^{v} / k_{B} T\right)\right)^{-1} .
$$

The next major advance was made by $\mathrm{Wu}$ [18 in 1966 who demonstrated, among other things, that the row correlation function $\left\langle\sigma_{0,0} \sigma_{0, N}\right\rangle$ behaves for $N$ large and $T<T_{c}$ as

$$
\left\langle\sigma_{0,0} \sigma_{0, N}\right\rangle \sim M_{-}^{2}\left\{1+C_{-}(T) \frac{\alpha_{2}^{2 N}}{N^{2}}+\cdots\right\}
$$

where

$$
\alpha_{2}=e^{-2 E^{v} / k_{B} T} \operatorname{coth}\left(E^{h} / k_{B} T\right)
$$

and

$$
C_{-}(T) \sim\left(T_{c}-T\right)^{-2} \quad \text { as } T \rightarrow T_{c},
$$

and for $T>T_{c}$ as

$$
\left\langle\sigma_{0,0} \sigma_{0, N}\right\rangle \sim M_{+}^{2} C_{+}(T) \frac{\alpha_{2}^{-N}}{N^{1 / 2}}
$$

where

$$
M_{+}=\left(1-k^{-2}\right)^{1 / 8}
$$

and

$$
C_{+}(T) \sim\left(T-T_{c}\right)^{-1 / 2} \quad \text { as } T \rightarrow T_{c} .
$$

The results $1.9-1.14$ have the property that if we let

$$
N \rightarrow \infty \text { and } T \rightarrow T_{c} \pm \text { with } r=N\left|T-T_{c}\right| \text { fixed }
$$

then the limits

$$
G(r)_{ \pm}=\lim M_{ \pm}^{-2}\left\langle\sigma_{0,0} \sigma_{0, N}\right\rangle
$$

exist and are non-zero. The limit 1.15 is called the scaling limit and the function (1.16) is called the scaling function. 
In 1976 these computations were extended by Wu, McCoy, Tracy and Barouch [19] to the general correlation function $\left\langle\sigma_{0,0} \sigma_{M, N}\right\rangle$ and not only were the leading terms computed but all terms were computed and the scaling function was computed for $T<T_{c}$ in the form

$$
G_{-}(r)=e^{F_{-}(r)} \quad \text { with } \quad F_{-}(r)=\sum_{n=1}^{\infty} F^{(2 n)}(r)
$$

and for $T>T_{c}$ as

$$
G_{+}(r)=X(r) e^{F_{-}(r)} \quad \text { with } \quad X(r)=\sum_{n=1}^{\infty} X^{(2 n-1)}(r)
$$

where $F^{(2 n)}(r)\left[X^{(2 n-1)}(r)\right]$ are given as $2 n[2 n-1]$ dimensional integrals.

But the truly remarkable result demonstrated in 19 is that these scaled twopoint functions are expressed in terms of the Painlevé III function defined as the solution of the equation

$$
\frac{d^{2} \eta}{d \theta^{2}}=\frac{1}{\eta}\left(\frac{d \eta}{d \theta}\right)^{2}-\frac{1}{\theta} \frac{d \eta}{d \theta}+\eta^{3}-\eta^{-1}
$$

with the boundary conditions

$$
\eta(\theta) \sim 1-2 \lambda K_{0}(2 \theta) \quad \text { as } \theta \rightarrow \infty \text { where } \lambda=1 / \pi,
$$

where $K_{0}(2 \theta)$ is the modified Bessel function, as

$$
G_{ \pm}(r)=\frac{1 \mp \eta(r / 2)}{\eta(r / 2)^{1 / 2}} \exp \int_{r / 2}^{\infty} d \theta \frac{1}{4} \theta \eta^{-2}\left[\left(1-\eta^{2}\right)^{2}-\left(\eta^{\prime}\right)^{2}\right] .
$$

The results which express the scaled two-point function of the Ising model in terms of a Painlevé function were published in physics journals [19, 1, 16] with titles that give no hint of the mathematics involved. However, because of his longstanding interest in physics, Sato read these papers and once he read them he realized that the Ising model had a far deeper connection with mathematics than anyone previously had any idea existed, and from 1977-1979 with Michio Jimbo and Tetsuji Miwa he developed, in a remarkable series of papers [12] and letters [13, the "theory of holonomic quantum fields".

In these papers on holonomic quantum field theory Sato recasts the Ising computations of [19] as a problem in quantum field theory and shows that the Green's functions of this theory satisfy maximally overdetermined (or holonomic) partial differential equations. Consequently, not only are the results of [19] recovered but the equations for all the $n$-point functions are obtained. None of this had been thought of or expected by the mathematics community and the developments are 
collected and presented in the papers 14, "Aspects of holonomic quantum fields. Isomonodromic deformation and Ising model. Complex analysis, microlocal calculus and relativistic quantum field theory" and [5], "The unanticipated link between deformation theory of differential equations and quantum fields".

\section{§2. The impenetrable Bose gas in one dimension}

Once Sato had invented holonomic quantum field theory he continued to apply it to other problems in physics. The first of these problems is the gas of impenetrable bosons in one dimension. The Hamiltonian of this system is the special case of $c \rightarrow \infty$ of

$$
H=-\frac{1}{2} \sum_{j=1}^{N} \frac{\partial^{2}}{\partial x_{j}^{2}}+c \sum_{k<j} \delta\left(x_{j}-x_{k}\right)
$$

which is equivalent to the quantum non-linear Schrödinger equation

$$
i \frac{\partial \phi}{\partial \phi}=\left[\phi, H_{c}\right]=-\frac{\partial^{2} \phi}{\partial x^{2}}+c \phi^{*} \phi^{2}
$$

with the commutation relation

$$
\left[\phi(x, t), \phi^{*}\left(x^{\prime}, t\right)\right]=\delta\left(x-x^{\prime}\right)
$$

and the Hamiltonian

$$
H_{c}=\frac{1}{2} \int_{0}^{L} d x\left(-\phi^{*} \frac{\partial^{2} \phi}{\partial x^{2}}+c \phi^{* 2} \phi^{2}\right) .
$$

The physical problem of interest for this system is the $n$-particle reduced density matrix at zero temperature defined as

$$
\begin{aligned}
& \rho_{n, N, L}\left(x_{1}, \ldots, x_{n} ; x_{1}^{\prime}, \ldots, x_{n}^{\prime}\right) \\
& =\frac{N !}{(N-n) !} \int_{0}^{L} \cdots \int_{0}^{L} d y_{n+1} \cdots d y_{N} \psi_{N, L}^{*}\left(x_{1}, \ldots, x_{n}, y_{n+1}, \ldots, y_{N}\right) \\
& \quad \times \psi_{N, L}\left(x_{1}^{\prime}, \ldots, x_{n}^{\prime}, y_{n+1}, \ldots, y_{N}\right)
\end{aligned}
$$

where $\psi_{N, L}\left(x_{1}, \ldots, x_{n}\right)$ is the $N$-particle ground state wave function normalized to unity $\left(\left\langle\psi_{N, L} \mid \psi_{N, L}\right\rangle=1\right)$. In particular we are interested in the limit $N, L \rightarrow \infty$ where

$$
\lim _{N, L \rightarrow \infty} \rho_{1, N, L}(x ; x)=\lim _{N, L \rightarrow \infty} N / L=\rho_{0} .
$$


Sato's study of this Bose gas begins with the study [4, 2] of the XY quantum spin chain defined by the Hamiltonian

$$
H_{X Y}=-\frac{1}{4} \sum_{j=-\infty}^{\infty}\left\{(1+\gamma) \sigma_{j}^{x} \sigma_{j+1}^{x}+(1-\gamma) \sigma_{j}^{y} \sigma_{j+1}^{y}+2 h \sigma_{j}^{z}\right\}
$$

where $\sigma_{j}^{i}$ are the Pauli spin matrices at site $j$ with

$$
\sigma^{x}=\left[\begin{array}{ll}
0 & 1 \\
1 & 0
\end{array}\right], \quad \sigma^{y}=\left[\begin{array}{rr}
0 & -i \\
i & 0
\end{array}\right], \quad \sigma^{z}=\left[\begin{array}{rr}
1 & 0 \\
0 & -1
\end{array}\right] .
$$

For arbitrary $\gamma \neq 0$ this quantum spin chain has a behavior at $h=1$ which is intimately related to the Ising model at $T=T_{c}$. However, there are most interesting new features which appear in the "double scaling" limit where $\gamma \rightarrow 0$ and $h \rightarrow 1-$ that are obtained by the methods of holonomic quantum field theory and deformation theory of differential equations. Furthermore there is an exact mapping of this double scaling limit to the impenetrable Bose gas problem and thus in a paper of fundamental importance 3. Sato and coauthors find differential equations which are satisfied by the $n$-particle reduced density matrix.

To illustrate these results we choose the normalization $\pi \rho_{0}=1$ and consider the one-particle density matrix

$$
\lim _{N, L \rightarrow \infty} \rho_{1, N, L}\left(x ; x^{\prime}\right)=\rho\left(x-x^{\prime}\right) .
$$

The result of [3] is that

$$
\rho(x)=\rho_{0} \exp \int_{0}^{x} d x^{\prime}\left(\frac{x^{\prime}}{4 y(1-y)^{2}}\left(\left(\frac{d y}{d x^{\prime}}\right)^{2}+4 y^{2}\right)-\frac{(1+y)^{2}}{4 x^{\prime} y}\right)
$$

where $y=y\left(x^{\prime}\right)$ satisfies the Painlevé $\mathrm{V}$ equation

$$
\begin{aligned}
\frac{d^{2} y}{d x^{2}}= & \left(\frac{1}{2 y}+\frac{1}{y-1}\right)\left(\frac{d y}{d x}\right)^{2}-\frac{1}{x} \frac{d y}{d x} \\
& +\frac{(y-1)^{2}}{x^{2}}\left(\alpha y+\frac{\beta}{y}\right)+\frac{\gamma y}{x}+\frac{\delta y(1+1)}{y-1}
\end{aligned}
$$

with $\alpha=1 / 2, \beta=-1 / 2, \gamma=-2 i, \delta=2$ and the boundary conditions

$$
\begin{aligned}
& y(x)=-1-\frac{2 i}{3} x+\left(\frac{2}{9}+\frac{2 i}{\pi}\right) x^{2}+O\left(x^{3}\right) \quad \text { as } x \rightarrow 0, \\
& y(x)=-e^{-2 i x}\left(1+O\left(x^{-1}\right)\right) \quad \text { as } x \rightarrow \infty .
\end{aligned}
$$

Furthermore if we set

$$
\sigma(x)=x \frac{d}{d x} \ln \rho(x)
$$


it is found that

$$
\left(x \frac{d^{2} \sigma}{d x^{2}}\right)^{2}=-4\left(x \frac{d \sigma}{d x}-1-\sigma\right)\left(x \frac{d \sigma}{d x}+\left(\frac{d \sigma}{d x}\right)^{2}-\sigma\right)
$$

with the boundary conditions

$$
\begin{array}{ll}
\sigma(x)=-\frac{1}{3} x^{2}+\frac{1}{3 \pi} x^{2}+O\left(x^{4}\right) \quad \text { as } x \rightarrow 0, \\
\sigma(x)=-\frac{1}{2}-\frac{1}{4 x} \sin 2 x+O\left(x^{-2}\right) & \text { as } x \rightarrow \infty,
\end{array}
$$

It is from these results for the Ising model and the delta function Bose gas that Sato [10, 15, 11] was able to make the profound mathematical generalization that the solutions of integrable classical soliton equations such as KdV and KP can be expressed as Plücker relations on an infinite-dimensional Grassmannian manifold.

\section{§3. Random matrices}

But perhaps the most important, influential and totally unexpected physics result of [3] is the pioneering discovery which is made in the theory of random matrices.

A random matrix is an $N \times N$ matrix whose matrix elements are chosen randomly out of a probability distribution. Physicists have been interested in the distribution of eigenvalues of random matrices starting with the work of Wigner [17. who was interested in random Hermitian matrices for a statistical description of energy levels for large nuclei. Wigner considered the level spacing probability $E(s)$ (where $s$ is the spacing between levels) of a Hermitian matrix whose matrix elements are chosen from a Gaussian distribution. The probability distribution is invariant under unitary transformations and this ensemble of matrices is called the Gaussian Unitary Ensemble (GUE). By means of approximate arguments Wigner [17] argued that this level spacing should be approximated in the limit $N \rightarrow \infty$ by

$$
E_{W}(s)=\frac{\pi s}{2} \exp \left(-\frac{\pi}{4} s^{2}\right)
$$

It would seem out of the question that the level spacing of such a random matrix could have anything to do with holonomic systems of partial differential equations which would seem to be the most constrained and least random of mathematical objects. It is therefore a stunning discovery, tucked away in a few lines in [3], that for the Gaussian unitary ensemble the level spacing is expressed 
as

$$
E(t)=\exp \int_{0}^{\pi t} d t^{\prime} \frac{\sigma\left(t^{\prime}\right)}{t^{\prime}}
$$

where $\sigma$ satisfies

$$
\left(x \frac{d^{2} \sigma}{d x^{2}}\right)^{2}=-4\left(x \frac{d \sigma}{d x}-\sigma\right)\left(x \frac{d \sigma}{d x}+\left(\frac{d \sigma}{d x}\right)^{2}-\sigma\right)
$$

with the boundary condition

$$
\sigma(x)=-\lambda x+O\left(x^{2}\right)
$$

with $\lambda=1 / \pi$. The equation (3.3) is equivalent to the Painlevé $\mathrm{V}$ equation (2.11) with $\alpha=\beta=0, \gamma=-2 i, \delta=2$.

This result for the level spacing of the Gaussian unitary ensemble is the key to dramatic advances in random matrix theory which have been made by C. A. Tracy and H. Widom in a long series of papers for which they won the Pólya prize of SIAM in 2002. There are two other ensembles, the Gaussian Orthogonal Ensemble and the Gaussian Symplectic Ensemble, which may also be studied by the methods of [3, and there are more refined properties of the spectrum which may also be obtained. Furthermore random matrix theory has found many applications to such diverse subjects as flame fronts and collisions of high energy particles which are far removed from the original motivation of Wigner in studying nuclear energy levels. Sato's work in [3] is a monument to what can be accomplished when incisive mathematical power is applied to problems of physical importance.

\section{$\S 4$. Algebraic analysis and the Sato school}

The contributions of Sato to mathematical physics do not stop with the discovery of the unexpected connection of the deformation theory of differential equations to problems in statistical mechanics and random matrix theory. Indeed, Sato's contribution is not limited to the papers he has written because Sato has the distinction of having founded an entire school of mathematical physics which is known as "algebraic analysis."

At first sight the term "algebraic analysis" appears to be self-contradictory because at the heart of analysis are limiting procedures whereas limits do not appear at all in algebra. So how can algebra and analysis coexist in the same subject??

The answer to this paradox is that every problem for which a physicist will say that there is an exact or closed form solution is actually controlled by an algebra. All of the results found by Sato which lead to Painlevé equations are the result of 
symmetry algebras and thus holonomic differential equations, which would seem to be in the province of analysis, are seen to actually be in the purview of algebra.

This deep appreciation of the role of algebra in the solution of problems in physics is the hallmark of what I will call the "Sato school" of mathematical physics as it has developed at the hands of Sato's former students Michio Jimbo and Tetsuji Miwa and their many collaborators and students. This has led to magnificent advances in representation theory and the invention of quantum groups which have had a transforming effect in mathematical physics from statistical mechanics to string theory. Sato's accomplishments are many and long lasting, and mathematical physics has been immensely enriched by his labors.

\section{References}

[1] E. Barouch, B. M. McCoy and T. T. Wu, Zero-field susceptibility of the two dimensional Ising model near $T_{c}$, Phys. Rev. Lett. 31 (1973), 1409-1411.

[2] M. Jimbo, T. Miwa, Y. Môri and M. Sato, Studies on holonomic quantum fields. XVI Density matrix of impenetrable Bose gas, Proc. Japan Acad. Ser. A Math. Sci. 55 (1979), 317-322. MR 0555056

[3] _ Density matrix of an impenetrable Bose gas and the fifth Painlevé transcendent, Phys. D 1 (1980), 80-158. Zbl 1194.82007 MR 0573370

[4] M. Jimbo, T. Miwa and M. Sato, Studies on holonomic quantum fields XV. Double scaling limit of one dimensional XY model, Proc. Japan Acad. Ser. A Math. Sci. 55 (1979), 267-272. MR 0553397

[5] M. Jimbo, T. Miwa, M. Sato and Y. Môri, Holonomic quantum fields. The unanticipated link between deformation theory of differential equations and quantum fields, in Mathematical problems in theoretical physics (Lausanne, 1979), Lecture Notes in Phys. 116, Springer, Berlin, 1980, 119-142. MR 0582615

[6] B. Kaufman, Crystal statistics II. Partition function evaluated by spinor analysis, Phys. Rev. 76 (1949), 1232-1243. Zbl 0035.42801

[7] B. Kaufman and L. Onsager, Crystal statistics III. Short range order in a binary Ising lattice, Phys. Rev. 76 (1949) 1244-1252. Zbl 0035.42802

[8] L. Onsager, Crystal statistics I. A two dimensional model with an order disorder transition, Phys. Rev. 65 (1944), 117-149. Zbl 0060.46001 MR 0010315

[9] _ discussion, Nuovo Cimento 6 Suppl. (1949), 261.

[10] M. Sato, Soliton equations as dynamical systems on infinite dimensional Grassmann manifolds, in Random systems and dynamical systems (Kyoto, 1981), RIMS Kokyuroku 439, Kyoto Univ., 1981, 30-46. Zbl 0507.58029 MR 0643105

[11] M. Sato, The KP hierarchy and infinite dimensional Grassmann manifolds, in: Theta functions-Boudoin 1987 (Brunswick, ME 1987), Proc. Sympos. Pure Math. 49, Part 1, Amer. Math. Soc., Providence, RI, 1989, 51-66. Zbl 0688.58016 MR 1013125

[12] M. Sato, T. Miwa and M. Jimbo, Holonomic quantum fields I-V, Publ. Res. Inst. Math. Sci. 14 (1978), 223-267; 15 (1979), 201-278, 577-629, 871-972; 16 (1980), 531-584. Zbl 0383.35066 (I), Zbl 0433.35058 (II), Zbl 0436.35076 (III), Zbl 0436.35077 (IV),

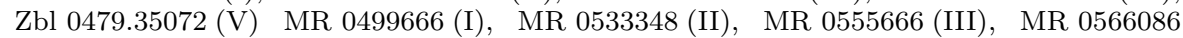
(IV), MR $0594916(\mathrm{~V})$ 
[13] Studies on holonomic quantum fields I-VIII, X-XII, Proc. Japan Acad. Ser. A Math. Sci. 53 (1977), 6-10, 147-152, 153-158, 183-185, 219-224; 54 (1978), 1-5, 36-41, 221225, 309-313; 55 (1979), 6-9, 73-77. $\quad$ MR 0674350 (I), $\quad$ MR 0674487 (II), $\quad$ MR 0674488

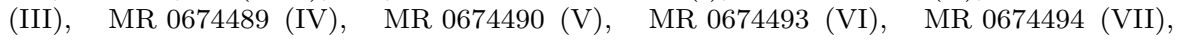
MR 0517325(VIII), MR 0520980 (X), $\quad$ MR 0525007 (XI), $\quad$ MR 0531446 (XII)

[14] - Aspects of holonomic quantum fields. Isomonodromic deformation and Ising model, in Complex analysis, microlocal calculus and relativistic quantum theory (Les Houches, 1979), Lecture Notes in Phys. 126, Springer, Berlin, 1980, 429-491. Zbl 0451.34008 MR 0579762

[15] M. Sato and Y. Sato, Soliton equations as dynamical systems on infinite-dimensional Grassmann manifold, in Nonlinear partial differential equations in applied science, North-Holland Math. Stud. 81, North-Holland, Amsterdam, 1983, 259-271. Zbl 0528.58020 MR 0730247

[16] C. A. Tracy and B. M. McCoy, Neutron scattering and the correlations functions of the Ising model near $T_{c}$, Phys. Rev. Lett. 31 (1973), 1500-1504.

[17] E. P. Wigner, Results and theory of resonance absorption, in Gatlinberg conference on neutron physics by time of flight, 1956, Oak Ridge Natl. Lab. Report ORNL-2309 (1957), 59-70; reprinted in Statistical theories of spectra: fluctuations, edited by C. E. Porter, Academic Press, New York, 1959.

[18] T. T. Wu, Theory of Toeplitz determinants and the spin correlations of the two dimensional Ising model I, Phys. Rev. 149 (1966), 380-401.

[19] T. T. Wu, B. M. McCoy, C. A. Tracy and E. Barouch, Spin-spin correlation functions for the two dimensional Ising model: exact theory in the scaling region, Phys. Rev. B 13 (1976), 316-374.

[20] C. N. Yang, The spontaneous magnetization of the two dimensional Ising model, Phys. Rev. 85 (1952), 808-816. Zbl 0046.45304 MR 0051740 